\title{
Goldston syndrome with congenital hepatic fibrosis: A rare cause of neonatal cholestasis
}

\author{
Shipra Agrwal ${ }^{1, *}$, Aashima Dabas ${ }^{1}$, Tej Pal ${ }^{2}$, Urmila Jhamb $^{1}$ \\ ${ }^{1}$ Department of Pediatrics, Maulana Azad Medical College, New Delhi, India; \\ ${ }^{2}$ Department of Radio diagnosis, Maulana Azad Medical College, New Delhi, India.
}

\begin{abstract}
Summary Goldston syndrome (GS) is a rare association of Dandy-Walker malformation (DWM) and cystic renal dysplasia with or without hepatic fibrosis. It is considered to be a milder variant of Meckel Gruber syndrome (MGS) and shares features with Miranda syndrome. We reported a 22 day old infant with DWM and autosomal recessive polycystic kidney disease (ARPKD) who presented with cholestasis and acholic stools. Ultrasonography and magnetic resonance cholangiopancreatography (MRCP) confirmed the diagnosis of congenital hepatic fibrosis (CHF). The child improved with supportive treatment. CHF is a rare condition which may present as a syndromic association.
\end{abstract}

Keywords: Dandy walker malformation, cystic renal disease, syndromic association, neonatal cholestasis, fibrocystic disease of liver

\section{Introduction}

Goldston syndrome (GS) was first described by Goldston in 1963 and is characterized by cystic renal dysplasia with Dandy-Walker malformation (DWM) with or without congenital hepatic fibrosis (CHF) (1). GS has been reported in antenatal cases and among few surviving babies postnatally (2-4). CHF is an autosomal recessive disorder caused by defective development of ductal plate during the embryonal period. It usually presents with features of portal hypertension in older children and adolescents. CHF may be associated with renal or central nervous system malformations as syndromic associations like Joubert syndrome, Bardet Biedl Syndrome and Meckel Gruber syndrome (MGS). GS is a rare cause of CHF.

The following record describes a rare case of a neonate with GS who was diagnosed with CHF with reported survival using conservative management.

Released online in J-STAGE as advance publication May 14, 2019.

*Address correspondence to:

Dr. Shipra Agrwal, Department of Pediatrics, Maulana Azad Medical College, Bahadurshah Zafar road, New Delhi 110002, India.

E-mail: shiprapaeds@gmail.com

\section{Case Report}

A male baby delivered at term, birth weight 2,750 grams, born of third degree consanguineous marriage presented at 22 days of life with fever, lethargy and jaundice noted for the last three days. Antenatal ultrasound in third trimester detected a large cystic area in posterior fossa communicating with fourth ventricle measuring $3.6 \times 4.0 \mathrm{~cm}$ with absent cerebellar vermis suggestive of DWM and bilateral enlarged and echogenic kidneys without oligohydramnios. The perinatal course of the baby was uneventful. Postnatal examination did not reveal any dysmorphism, hepatosplenomegaly, external cranial defects, eye abnormality or any cardiac anomaly. Head circumference was normal at birth (35 $\mathrm{cm})$. Ultrasonography on day two of life confirmed the presence of DWM and showed bilateral enlarged $(6.3 \times 3.1 \mathrm{~cm}$ and $6.0 \times 3.1 \mathrm{~cm})$ kidneys with increased cortical echogenicity and tiny multiple cysts suggestive of autosomal recessive polycystic kidney disease (ARPKD). There were small cysts in left liver lobe with mild dilatation of intrahepatic biliary radicals. Baby was discharged on day 18 of life on exclusive breastfeeding until he became sick. There was no history of seizures, bleeding manifestations, abdominal distension, edema or decreased urine output. The previous pregnancy was terminated at 18 weeks gestation due to antenatal detection of a central nervous system malformation. 


\section{Autopsy was not done.}

At presentation child was lethargic, icteric, febrile with heart rate of 140/min and respiratory rates of 55/ min with normal oxygen saturation and weight of 3,100 grams. Head circumference had increased by five $\mathrm{cm}$ to $40 \mathrm{~cm}$. Blood pressure was normal. There was no pallor or edema. There was firm hepatomegaly (span $8 \mathrm{~cm}$ ) and splenomegaly (1 cm enlarged). Three consecutive stool samples were observed and found to be acholic. His hemoglobin was $145 \mathrm{gm} / \mathrm{L}$, total leucocyte count was $10.1 \times 10^{9} / \mathrm{L}$ (lymphocytes $65 \%$ ) and normal platelets. Liver function tests showed total bilirubin of $177.8 \mu \mathrm{mol} / \mathrm{L}$ (direct $76.9 \mu \mathrm{mol} /$ L), aspartate and alanine aminotransferase as 364 and $789 \mathrm{IU} / \mathrm{L}$ respectively. Total protein was $50 \mathrm{~g} / \mathrm{L}$ with serum albumin of $35 \mathrm{~g} / \mathrm{L}$, prothrombin time and INR were normal. Alkaline phosphatase was $902 \mathrm{IU} / \mathrm{dL}$ and gammaglutamyl transferase (GGT) was 16.34 ukat/L. The renal functions, serum electrolytes, blood glucose and chest X-ray were normal. Blood and urine cultures were sterile.

A possibility of cholangitis or sepsis induced cholestasis was considered and child was started on intravenous fluids, injection cefotaxime and amikacin and supplements for cholestasis. His fever and general condition improved after 5 days, he became afebrile and started accepting breast feeding. His stools also became pigmented. Ultrasound of the abdomen showed a distended gall bladder with normal post feed contractility, normal bile duct, altered echotexture with increased echogenicity along the portal tracts in liver parenchyma. Bilateral kidneys were enlarged with multiple tiny cysts suggestive of ARPKD. A provisional diagnosis of CHF (with polycystic kidney disease and CNS malformation) was made. Magnetic resonance cholangiopancreatography (MRCP) showed

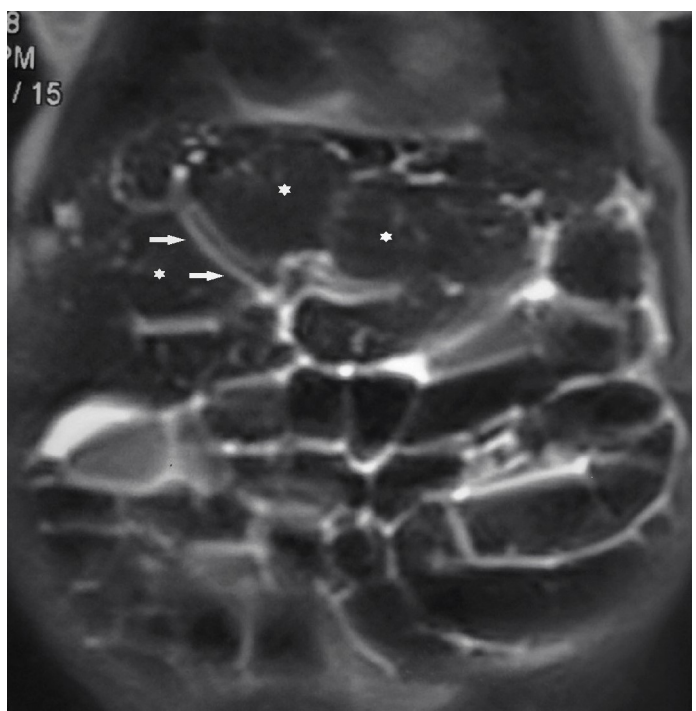

Figure 1. Coronal T2 weighted magnetic resonance cholangiopancreatography showing diffusely hypo intense liver parenchyma (asterisk) and periportal T2 hyper intensities (arrows). diffusely hypointense liver with linear T2 hyper intense bands along the portal tracts with normal biliary tract suggestive of CHF (Figure 1), diffusely enlarged bilateral kidneys with increased $\mathrm{T} 2$ signal intensity and loss of corticomedullary differentiation suggestive of ARPKD (Figure 2). Liver biopsy was not performed. Magnetic resonance imaging (MRI) of brain showed large cyst in posterior fossa, which was communicating with fourth ventricle, with absence of cerebellar vermis suggestive of DWM (Figure $3 \mathrm{~A}$ and $3 \mathrm{~B}$ ). The syndromic association of CHF, ARPKD and DWM was evaluated and possibility of GS was considered.

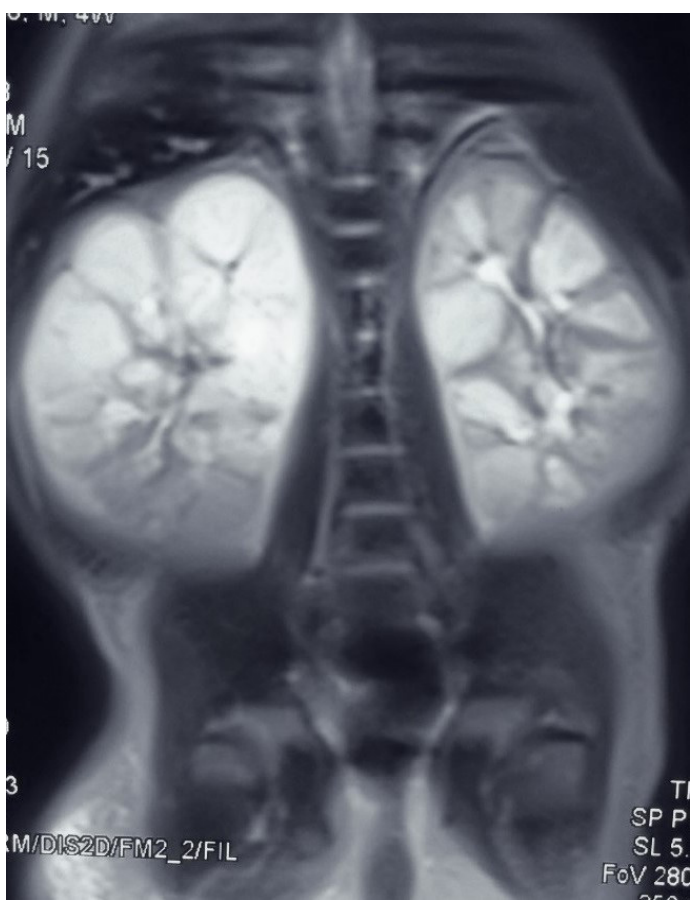

Figure 2. Coronal T2 weighted magnetic resonance imaging of abdomen showing enlarged bilateral kidneys with increased T2 signal intensity and loss of corticomedullary differentiation.
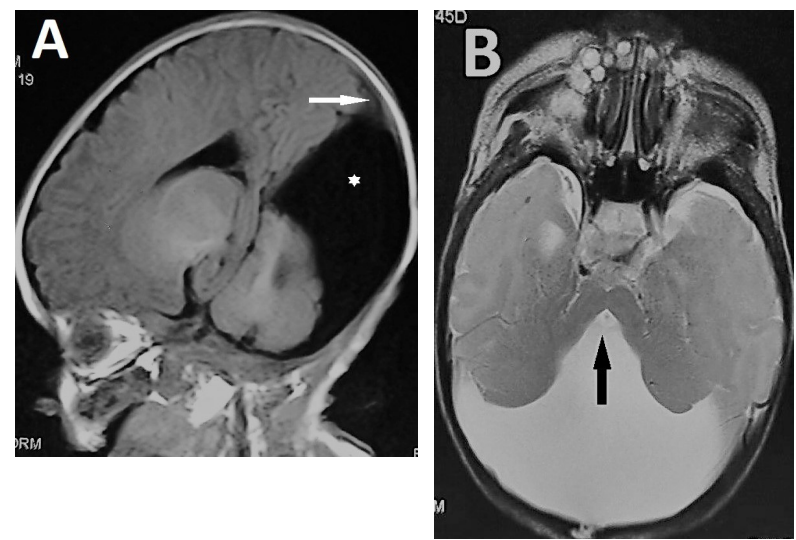

Figure 3. Magnetic resonance imaging of the brain. (A), T2 FLAIR sagittal section showing superior displacement of torcular herophili (arrow) with cystic dilatation of fourth ventricle (asterisk); (B), T2 weighted coronal section showing cystic dilatation of fourth ventricle with absence of cerebellar vermis (arrow). 
The child was continued on supportive conservative treatment, breastfeeding, and supplements on which his jaundice improved. A cystoperitoneal shunt is planned for DWM.

\section{Discussion}

GS is a rare syndrome initially detected in antenatal life with constellation of DWM and renal abnormalities (13). Moerman et al. reported two surviving siblings with cystic renal dysplasia, central nervous system (CNS) abnormality with cranium bifidum and hepatic ductal plate malformation. One of them had DWM while the other had occipital encephalocoele. They proposed that GS was a milder variant of MGS based on similar findings seen in both syndromes (5). Another familial series was reported by Walpole et al. of three siblings with DWM associated with cystic renal dysplasia. CHF was seen in one of these siblings and none of them had polydactyly. They concluded it to be a syndrome distinct from MGS owing to absence of cardinal features of MGS apart from cystic renal dysplasia (6). There are other rare reports of subjects with GS who had survived beyond the perinatal period $(4,7)$. The index case is one of the rare cases surviving beyond neonatal period. Although hepatic fibrosis has been previously reported, there is no reported case of cholestasis in neonate as a manifestation of GS as seen in the index case. Other reported features of GS include facial dysmorphism, hypoplasia of carpus callosum and absence of spleen $(4,6)$. There is familial association involving both male and female suggesting an autosomal recessive (AR) inheritance. Presence of consanguinity and family history in the index case points to AR inheritance. The previous aborted sibling of index case also had brain abnormality presumed to be a similar syndromic association.

The prototype syndrome involving central nervous system and kidneys is MGS. MGS is an autosomal recessive syndrome characterized by cystic renal dysplasia, occipital encephalocoele and post axial polydactyly affecting all four limbs (8). All three cardinal features are found in only about half of them (1). Two of these three anomalies are required to make the diagnosis of MGS (9). The incidence ranges from one in 13,000$4,00,000$ (10). A variety of other malformations have been reported in MGS. MGS may be associated with agenesis of cerebellar vermis and posterior fossa cyst suggestive of DWM, cystic hygroma, cardiac malformation, microcephaly, IUGR, and cleft palate (9,11-13). Hepatic fibrosis and bile duct proliferation has also been reported and can be present in up to $1 / 5$ th of the cases $(4,14)$. Cystic renal dysplasia is the most constant feature while post axial polydactyly is the most variable feature of MGS $(9,11)$. Most babies are either still birth, medically terminated or die soon after birth (15). The index case did not qualify for MGS due to absence of occipital encephalocoele and polydactyly. Another rare syndrome similar to GS is Miranda syndrome, also known as cerebrohepatorenal syndrome, which is characterized by cystic renal dysplasia, DWM and hepatic fibrosis. It is believed that both GS and Miranda syndrome are milder variants of MGS (1). Another syndrome associated with DWM and hepatic fibrosis is $\mathrm{COACH}$ syndrome with additional features like ataxia, nystagmus, oculomotor apraxia, developmental delay and facial dysmorphism. Gentile et al reported two siblings with this syndrome, one of them required liver transplantation due to liver failure caused by CHF (16). The index case differs due to presence of renal involvement and absence of other features of $\mathrm{COACH}$ syndrome.

$\mathrm{CHF}$ is an autosomal recessive fibrocystic disease of the liver. It results from failure of remodeling of the ductal plates during embryonic development leading to persistence of immature duct structures which stimulate hepatic stellate cells to cause progressive periportal fibrosis. Patients commonly present in adolescence or adulthood with features of portal hypertension and cytopenias due to hypersplenism with preserved hepatic synthetic function. In a study on 26 patients with $\mathrm{CHF}$ with mean age of 28.4 years, most common symptom was abdominal distension with splenomegaly (43\%) followed by recurrent cholangitis $(23 \%)$ and esophageal variceal bleed (7.7\%) (17). Another study involving 25 patients with CHF (mean age 8.5yr) reported hematemesis (60\%) and abdominal distension (48\%) as common symptoms with 5 (20\%) of cases each detected incidentally and on sibling screening (18). Presentation of CHF as neonatal cholestasis is rare (19). The index case presented during neonatal period with features of cholangitis and improved with supportive management. Typical hepatic imaging findings included caudate lobe hypertrophy, periportal T2 hyper intensities and biliary duct anomalies in cases with Caroli syndrome (20). Liver biopsy was not done in the index case but MRCP findings in association with ARPKD were diagnostic of CHF. CHF is associated with many syndromes and common ones being Joubert syndrome, Bardet Biedl syndrome, and MGS (17). Goldston and Miranda syndrome are also associated with $\mathrm{CHF}$.

To conclude, we describe a rare presentation of GS presenting in neonatal period with cholestasis confirmed to be CHF. Determining the syndromic association is important for holistic management and long term prognosis.

\section{Acknowledgements}

We thank the parents of the patient for allowing us to publish the information about their child that will help increase knowledge about the disease.

\section{References}

1. Avcu S, Akdeniz H, Unal O, Kurdoğlu M. Goldston 
syndrome in a fetus: Case report and literature review. Fetal Pediatr Pathol. 2010; 29:353-358.

2. Gulcan YH, Duman N, Kumral A, Sağol, Lebe B, Kavukçu S, Erçal D, Celiloğlu M, Ozkan H. Goldston syndrome: Report of a case. Genet Couns. 2001; 12:263267.

3. Gloeb DJ, Valdes-Dapena M, Salman F, O'Sullivan MJ, Quetel TA. The Goldston syndrome: Report of a case. Pediatr Pathol. 1989; 9:337-343.

4. Hussain Z, Masroor I, Haider Q, Alam T. Goldston syndrome. J Coll Physicians Surg Pak. 2011; 21:242-244.

5. Moerman P, Pauwels P, Vandenberghe K, Lauweryns JM, Fryns JP. Goldston syndrome reconsidered. Genet Couns. 1993; 4:97-102.

6. Walpole IR, Goldblatt J, Hockey A, Knowles S. DandyWalker malformation (variant), cystic dysplastic kidneys, and hepatic fibrosis: A distinct entity or Meckel syndrome? Am J Med Genet. 1991; 39:294-298.

7. Menon RK, Nadkarni TD, Desai KI, Goel A. DandyWalker malformation associated with polycystic kidneys: Goldston syndrome revisited. J Clin Neurosci. 2006; 13:875-877.

8. Gupta M, Mehta A, Gupta R, Gupta R, Singh S. Prenatal diagnosis of Meckel-Gruber syndrome with Dandy Walker malformation. JK Science. 2005; 7:164-166.

9. Alam A, Adhi M, Bano R, Zubair A, Mushtaq A. Meckel Gruber Syndrome: Second trimester diagnosis of a case in a non-consanguineous marriage. Pak J Med Sci. 2013; 29:234-236,

10. Celentano C, Prefumo F, Liberati M, Gallo G, Di Nisio Q, Rotmensch S. Prenatal diagnosis of Meckel-Gruber syndrome in a pregnancy obtained with ICSI. J Assist Reprod Genet. 2006; 23:281-283.

11. Nyberg DA, Hallesy D, Mahony BS, Hirsch JH, Luthy DA, Hickok D. Meckel- Gruber syndrome. Importance of prenatal Diagnosis. J Ultrasound Med. 1990; 9:691696.
12. Balci S, Tekșen F, Dökmeci F, Cengiz B, Cömert RB, Can B, Ozdamar S. Prenatal diagnosis of Meckel-Gruber syndrome and Dandy-Walker malformation in four consecutive affected siblings, with the fourth one being diagnosed prenatally at 22 weeks of gestation. Turk J Pediatr. 2004; 46:283-288.

13. Aslan K, Külahçı Aslan E, Orhan A, Atalay MA. Meckel Gruber syndrome, A case report. Organogenesis. 2015; 11:87-92.

14. Su SL, Liu CM, Lee JN. Prenatal diagnosis of MeckelGruber syndrome case reports. Gaoxiong Yi Xue Ke Xue Za Zhi. 1995; 11:127-132. (in Chinese)

15. Gazioglu N, Vural M, Seckin MS, Tuysuz B, Akpir E, Kuday C, Ilikkan B, Erginel A, Cenani A. Meckel-Gruber syndrome. Childs Nerv Syst. 1998; 14:142-145.

16. Gentile M, Di Carlo A, Susca F, Gambotto A, Caruso ML, Panella C, Vajro P, Guanti G. COACH Syndrome: Report of two brothers with congenital hepatic fibrosis, cerebellar vermis hypoplasia, oligophrenia, ataxia, and mental retardation. Am J Med Genet. 1996; 64:514-520.

17. Shorbagi A, Bayraktar Y. Experience of a single center with congenital hepatic fibrosis: A review of the literature. World J Gastroenterol. 2010; 16:683-690.

18. Parkash A, Cheema HA, Malik HS, Fayyaz Z. Congenital hepatic fibrosis: Clinical presentation, laboratory features and management at a tertiary care hospital of Lahore. J Pak Med Assoc. 2016; 66:984-988.

19. Bharani V, Venkatesh GV, Saikia UN, Thapa BR. Congenital hepatic fibrosis with polycystic kidney disease: An unusual cause of neonatal cholestasis. Indian Pediatr. 2017; 54:589-593

20. Sureka B, Rastogi A, Bihari C, Bharathy KG, Sood V, Alam S. Imaging in ductal plate malformations. Indian J Radiol Imaging. 2017; 27:6-12.

(Received February 2, 2019; Revised April 9, 2019; Accepted May 3, 2019) 